\title{
Can short time delays influence the variability of the solar cycle?
}

\author{
Laurène Jouve, Michael R. E. Proctor and Geoffroy Lesur
}

DAMTP, Centre for Mathematical Sciences, Wilberforce Road, CB3 0WA CAMBRIDGE, UK

\begin{abstract}
We present the effects of introducing results of 3D MHD simulations of buoyant magnetic fields in the solar convection zone in 2D mean-field Babcock-Leighton models. In particular, we take into account the time delay introduced by the rise time of the toroidal structures from the base of the convection zone to the solar surface. We find that the delays produce large temporal modulation of the cycle amplitude even when strong and thus rapidly rising flux tubes are considered. The study of a reduced model reveals that aperiodic modulations of the solar cycle appear after a sequence of period doubling bifurcations typical of non-linear systems. We also discuss the memory of such systems and the conclusions which may be drawn concerning the actual solar cycle variability.
\end{abstract}

Keywords. Magnetic fields - Sun: activity - Sun: interior - Methods: numerical

\section{Introduction: time delay dynamics and solar dynamo models}

In the framework of mean-field dynamo theory, several possibilities have been studied to explain the variability of the solar cycle. They mainly fell into two categories: stochastic forcing or dynamical nonlinearities. Indeed, the solar convection zone is highly turbulent and it would be surprising if the dynamo processes acting inside this turbulent plasma were nicely regular. The influence of stochastic fluctuations in the meanfield dynamo coefficients has been studied in various models (e.g. Hoyng 1988, Weiss \& Tobias 2000, Charbonneau \& Dikpati 2000). Moreover, the dynamical feedback of the strong dynamo-generated magnetic fields is likely to be significant enough to produce non-linear effects on the activity cycle. A number of models have introduced these nonlinear effects (Tobias 1997, Moss \& Brooke 2000, Bushby 2006, Rempel 2006) and have resulted in the production of grand minima-like periods or other strong modulations of the cyclic activity. However, time delays have hardly been considered, even if they were known to increase the order of the governing equations and thus likely to produce interesting long-term evolutions.

Yoshimura (1978) was the first to introduce time delays both on the toroidal and poloidal potential equations. His values were quite arbitrary but he made the point that those time delays alone could act to produce long-term modulation of the periodic solutions. In Babcock-Leighton flux-transport models, a meridional flow is introduced and flux emergence from the base of the convection zone to the surface is implicitly present. In this particular model, the introduction of time delays and their consequences have also been studied (e.g. Wilmot-Smith et al. 2006) but the delays were mainly due to the advection time by meridional flow. Indeed, the time-scale of the buoyant rise of flux tubes was considered to be so small compared to the cycle period (and to the meridional flow turnover time) that this particular step was assumed to be instantaneous. However, we address here the question of the influence of magnetic field dependent delays on the cycle produced by Babcock-Leighton models and especially on its potential modulation. 


\section{What about the rise time of buoyant magnetic fields?}

\subsection{The modified source term for poloidal field}

In Babcock-Leighton dynamo models, the poloidal field owes its origin to the tilt of magnetic loops emerging at the solar surface. Since these emerging loops are thought to rise from the base of the convection zone through magnetic buoyancy, we see that we can directly relate the way we model the Babcock-Leighton (BL) source term and the results of 3D calculations of Jouve \& Brun (2009).

In the standard model, the source term is confined in a thin layer at the surface and is made to be antisymmetric with respect to the equator, due to the sign of the Coriolis force which changes from one hemisphere to the other. These features are retained in our modified version.

However, the standard term is proportional to the toroidal field at the base of the convection zone at the same time, implying an instantaneous rise of the flux tubes from the base to the surface where they create tilted active regions. The $3 \mathrm{D}$ calculations showed that the rise velocity and thus the rise time depend on the field strength at the base of the $\mathrm{CZ}$, we thus introduce in our modified version of the source term, a magnetic fielddependent time delay in the toroidal field at the base of the convection zone. We thus take into account the time delay between the formation of strong toroidal structures at the base of the convection zone and the surface regeneration of poloidal field.

The modified expression of the source term is thus

$$
\begin{aligned}
S\left(r, \theta, B_{\phi}\right)= & \frac{1}{2}\left[1+\operatorname{erf}\left(\frac{\mathrm{r}-\mathrm{r}_{2}}{\mathrm{~d}}\right)\right]\left[1-\operatorname{erf}\left(\frac{\mathrm{r}-1}{\mathrm{~d}}\right)\right] \times\left[1+\left(\frac{\mathrm{B}_{\phi}\left(\mathrm{r}_{\mathrm{c}}, \theta, \mathrm{t}-\tau_{\mathrm{B}}\right)}{\mathrm{B}_{0}}\right)^{2}\right]^{-1} \\
& \times \cos \theta \sin \theta B_{\phi}\left(r_{c}, \theta, t-\tau_{B}\right)
\end{aligned}
$$

where $r_{c}=0.7, r_{2}=0.95, d=0.01, B_{0}=10^{4}$, with the time delay $\tau_{B}$ proportional to the inverse of the magnetic energy at the base of the convection zone

$$
\tau_{B}(\theta, t)=\tau_{0} / B_{\phi}\left(r_{c}, \theta, t\right)^{2}
$$

In the $3 \mathrm{D}$ simulations, the approximate rise time for a $6 \times 10^{5} \mathrm{G}$ field is about 4 times that of a $3 \times 10^{5} \mathrm{G}$ field, agreeing with the formulation 2.2. This takes into account the more significant effects of convective downdrafts and Ohmic diffusion in the "weak B" case. Note that the time delay is then dependent both on space and time.

Finally, a threshold has been introduced on the source term for both very strong (above $10^{4} \mathrm{G}$ ) and very weak fields (below $100 \mathrm{G}$ ). We thus take into account the fact that very strong toroidal structures are not influenced by the Coriolis force enough to gain a significant tilt as they reach the surface and we prevent unrealistically large delays to appear in our model.

\subsection{Long-term modulation of the cycle amplitude}

The main result of our calculations (as shown in Jouve, Proctor \& Lesur 2010) is that increasing the time delays in our model tends to produce a long-term modulation of the magnetic activity, even in the limit of small delays compared to the full cycle. Figure 1 illustrates the behaviour of the toroidal magnetic energy at the base of the convection zone and at the latitude of $25^{\circ}$ when the delay is increased from 0 to 14 days on $5 \times 10^{4} \mathrm{G}$ fields. The major effect of the delays is to modulate the amplitude of the cycle to such a point that the weakest cycle on the particular period calculated here reaches in terms 

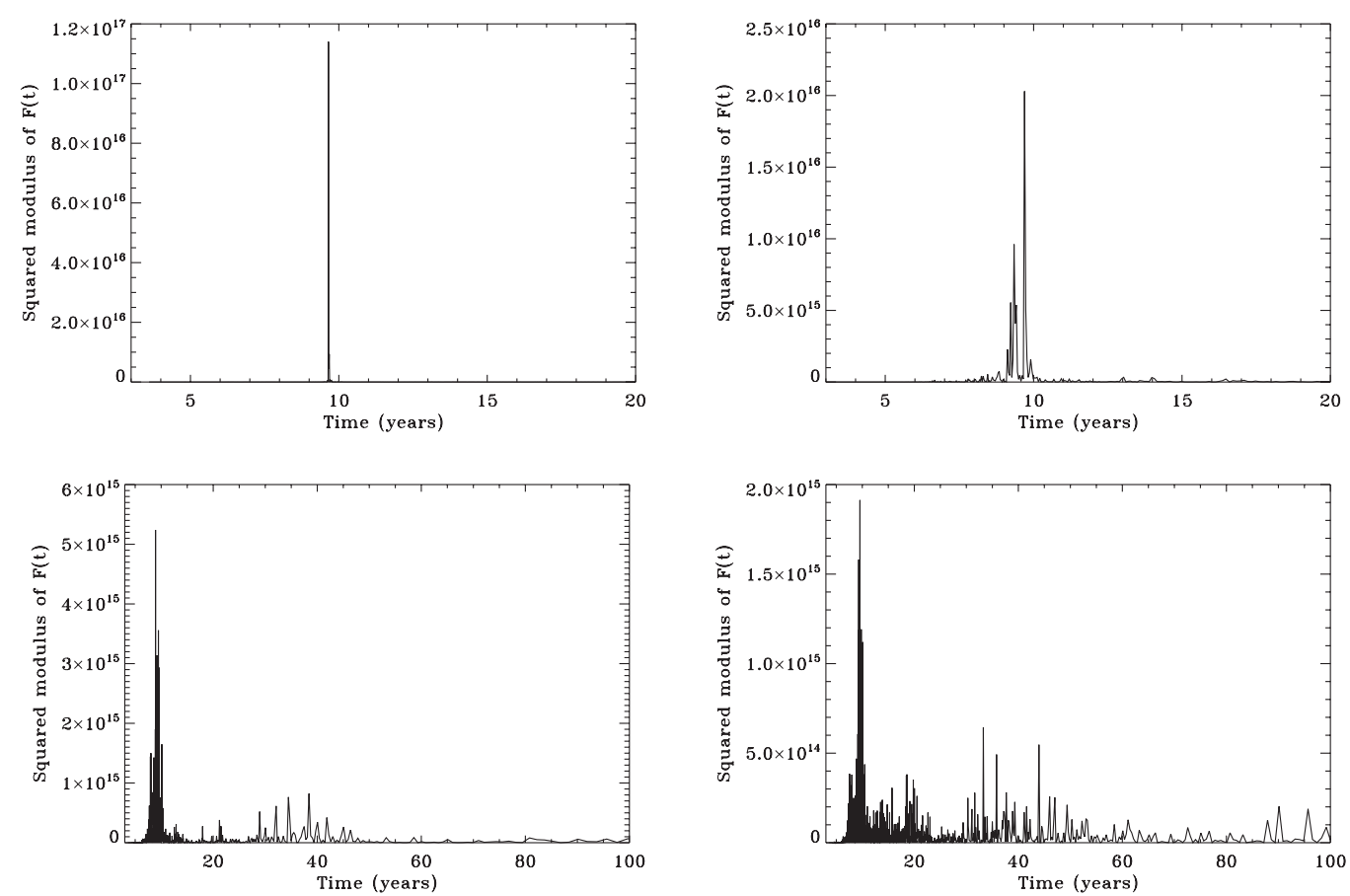

Figure 1. Powerspectra for the following values of the delay: no delay, 14 days delay on $5 \mathrm{kG}$-fields, on $10 \mathrm{kG}$-fields and on $50 \mathrm{kG}$-fields. The peak at $\mathrm{T} \approx 10$ years is clearly visible in every panels, the only difference is the appearance of extra frequencies for increasing delays. Note the change of scale for the abscissa in the two lower panels.

of peak energy only $30 \%$ of the peak energy of the strongest cycle. If we consider the powerspectra of the various cases (shown on Fig. 1), we can study if any particular secondary frequencies are excited and how this depends on the delay. The first striking point which can be noted from analysing this figure is that the fundamental cycle period is retained in all the models. The most significant peak is indeed always located at about 10 years. More interestingly, some additional periods seem to appear, particularly at higher values than the fundamental component. This shows our small time delays produce modulations of the cycle on time scales much longer than the cycle period. In particular, the last panel of Fig. 1 seems to show some secondary periods between 30 and 50 years, as well as a peak around 20 years, possibly resulting from a previous period doubling which led to this chaotic behaviour.

\subsection{A reduced model showing the route to chaos}

In Jouve, Proctor \& Lesur (2010), we develop a reduced model to make a tentative explanation of this observed modulated activity and the chaotic solution we obtain in the limit of strong delays. We summarize here the formulation of the model and the associated results.

The reduced model we choose to study does not contain any explicit delays, it rather introduces a new variable $Q_{t}$ which will represent the delayed toroidal field and will be responsible for the regeneration of the poloidal component. For simplification, the equations are written in one dimension and we use a Fourier expansion in this direction, with wavenumber $k=1$. We thus get the following set of complex ODEs: 


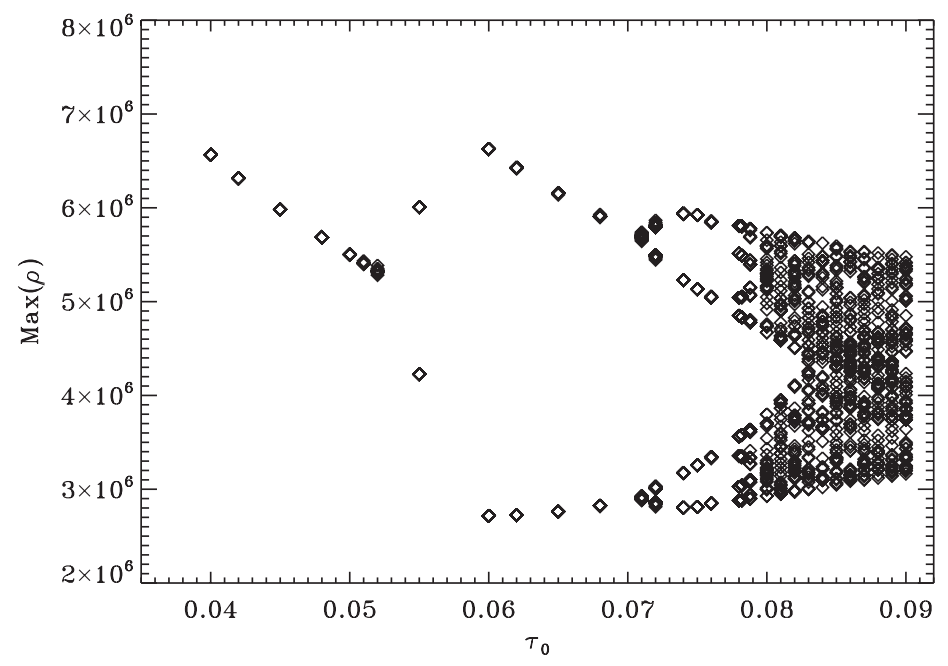

Figure 2. Bifurcation diagram obtained with a reduced model of the delayed equations. The maximum value of the modulus of $B_{t}$ is plotted here against the intensity of the delay.

$$
\begin{gathered}
\frac{d A_{t}}{d t}+i v A_{t}=\frac{S Q_{t}}{1+\lambda\left|Q_{t}\right|^{2}}-\eta A_{t} \\
\frac{d B_{t}}{d t}+i v B_{t}=i \Omega A_{t}-\eta B_{t} \\
\frac{d Q_{t}}{d t}=\frac{1}{\tau}\left(B_{t}-Q_{t}\right)
\end{gathered}
$$

with $\tau=\tau_{0} /\left(1+\left|B_{t}\right|^{2}\right)$.

Thanks to its phase invariance, this system can be further reduced from 6 to 5 degrees of freedom and the evolution of our solution can now be accurately followed when the delay is increased.

On Fig. 2, the peak amplitude of the modulus of $B_{t}$ is followed for successive discrete values of the delay. This procedure results in a bifurcation diagram showing a succession of period doublings leading to a chaotic behaviour when the delay on the strongest fields represents about $10 \%$ of the cycle period. Up till now, the nonlinearities at the origin of the sequence of bifurcations in most models were always related to the dynamical feedback of the Lorentz force on the flow. The striking result here is that the modulation of the cycle amplitude can also arise in models where the only nonlinearities are the quenching term in the Babcock-Leighton source and the magnetic field-dependent time delays. It has here to be noted though that the time delays also appear in the quenching term, possibly amplifying the nonlinear effects in the model.

\subsection{Memory of the system}

In this subsection, we go back to the full spherical $2 \mathrm{D}$ model and study the so-called memory of the system between various cycles. This analysis has been shown to be efficient at distinguishing between advection-dominated and diffusion-dominated models as discussed in Yeates, Nandy \& Mackay (2008). In their work, the peak surface radial flux for cycle $n$ is compared to the peak toroidal flux for cycles $n, n+1, n+2$ and $n+3$. 

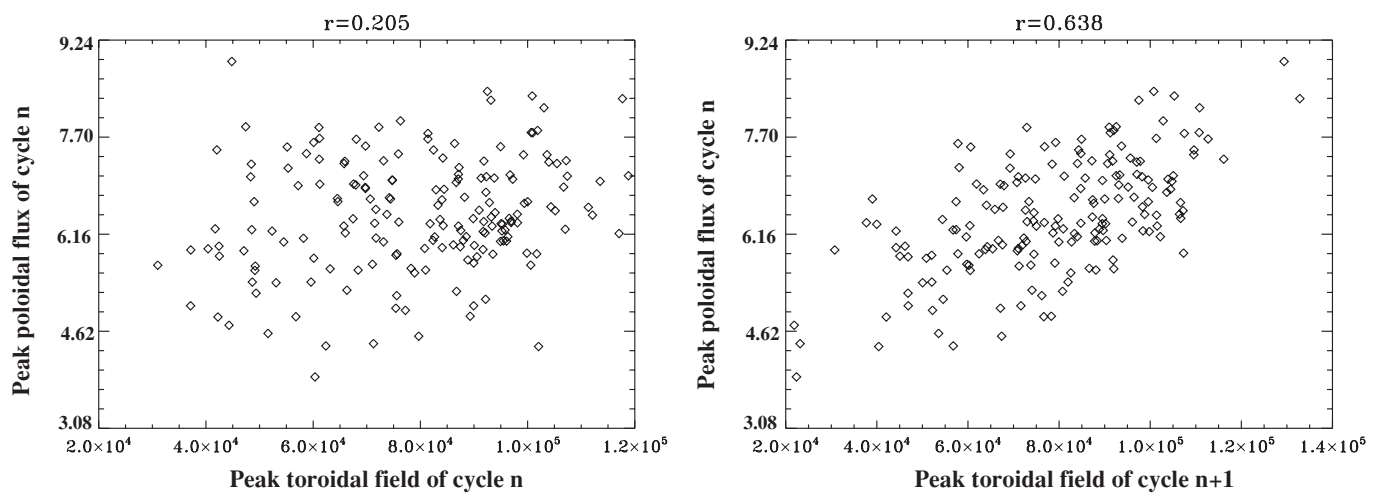

Figure 3. Correlation analysis between successive cycles. Only the relations between cycles $\mathrm{n} / \mathrm{n}$ (left panel) and $\mathrm{n} / \mathrm{n}+1$ (right panel) are shown here. The poloidal flux unit is $10^{24} \mathrm{Mx}$.

They find that in an advection-dominated model where the BL source term is stochastically perturbed, the fixed time delay imposed by the advection by the meridional flow is so dominant that the memory of the system persists for several cycles.

On the contrary, in the diffusion-dominated regime, the transport of magnetic fields by diffusion may act to short-circuit the conveyor belt related to the meridional flow profile. The time-scale of the meridional flow is thus less dominant and the only visible correlation is between cycles $n$ and $n+1$. We note here that the peak toroidal field precedes the peak surface radial field for the same cycle, which has the same sign. The poloidal field then produces the toroidal field for cycle $n+1$ with the opposite sign. It is thus natural to find a strong correlation between cycles $n$ and $n+1$ since it relies on the regeneration of toroidal fields by the differential rotation, a very robust (and unperturbed) effect.

In our model, it might also be interesting to look at the correlation between successive cycles. Indeed, we are still in the advection-dominated regime but we have now introduced a new time-scale by imposing our delays.

On Fig. 3, we plot the peak poloidal flux at the surface at high latitudes (between $60^{\circ}$ and $90^{\circ}$ ) against the unsigned peak toroidal field at the base of the convection zone at low latitudes (between 0 and $40^{\circ}$ ). The linear Pearson correlation coefficient has been computed for the relation between the poloidal component of cycle $n$ and the toroidal field of cycle $n, n+1, n+2$ and $n+3$. Figure 3 represents the relations between $n$ and $n$ and $n$ and $n+1$. The only significant correlation we find is between cycles $n$ and $n+1$ but is much less significant between cycle $n$ and the later ones. Indeed, the correlation coefficient is about 0.64 for cycles $n$ and $n+1$ and drops to values around 0.1 and 0.2 for the other cycles. The strong relation between cycles $n$ and $n+1$ is again explained by the robust $\Omega$-effect shearing the poloidal flux of cycle $n$ into the toroidal field of opposite sign of cycle $n+1$. This mechanism has not been modified by the introduction of time-delays and the correlation is thus naturally retained. On the contrary, the chaotic behaviour of our magnetic field prevents the memory of the system to persist for several cycles. After the toroidal field of cycle $n+1$ has been generated, the flux emergence producing the new poloidal field (which is then responsible for the toroidal field of cycle $n+2$ ) is perturbed by the time delays and the correlation between cycle $n$ and $n+2$, which was very significant in the advection-dominated regime, is mostly lost. We thus conclude that advection-dominated models may well lose their memory in the same way as the diffusion dominated ones, provided that additional time scales coexist with the typical time scale of the meridional flow (which is typically 2 to 3 cycles). 

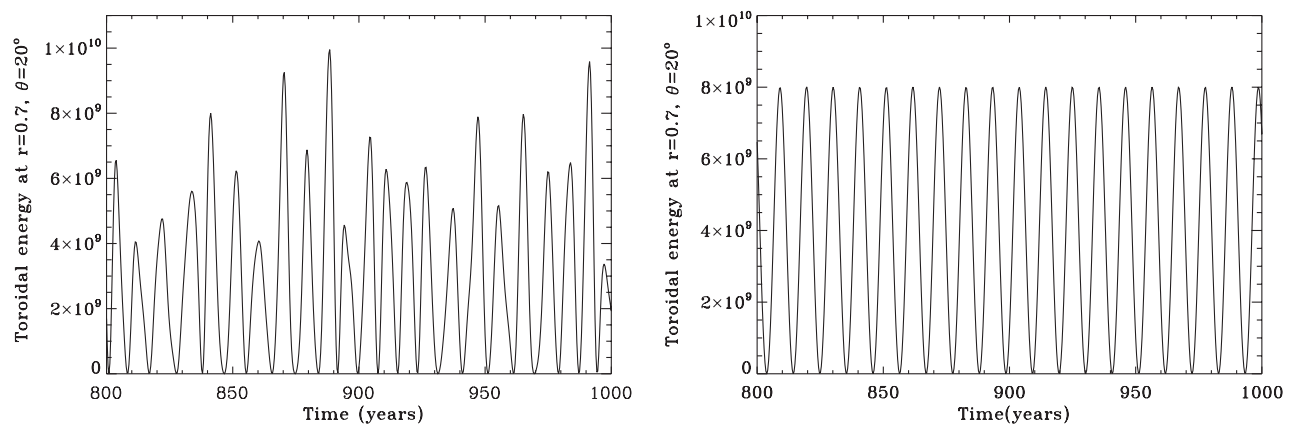

Figure 4. Toroidal energy at a particular point in space for a model with magnetic field-dependent delays and for a calculation where the delay has been fixed to about 1 year.

\section{Variations on the 2D model: stabilising effects?}

In this section, we try to point out the main factors responsible for the modulation of the cycle. The reduced model helped us to gain some insight on the mechanisms leading to a chaotic behaviour, we now turn to investigate the similarities with the $2 \mathrm{D}$ model in terms of stabilising or destabilising effects.

\subsection{Fixed vs varying delays}

The striking results of a long-term modulation due to small time-delays compared to the cycle period can be partly explained by the dependence of the delays on the magnetic field strength. Indeed, in the reduced model, it is clear that small but varying delays produce long-term modulation much sooner than fixed delays. The same feature is recovered in the 2D model. Figure 4 shows the time evolution of the toroidal magnetic field at a particular point in space in a case where the delay varies according to Eq. 2.2 and in a case where the delay is fixed to about 1 year for any field strength. It is clear that a fixed delay about one order of magnitude smaller than the cycle period has a very weak effect on the long-term activity, as expected. On the contrary, when the delay is made to vary with the field strength, reaching values between 0 and 5 years, the effect on the long-term modulation is much stronger, as shown on the first panel of Fig. 4. This particular case corresponds to the last panel of Fig. 1 which illustrates the existence of various dominant frequencies in the time series of the same toroidal field.

If the fixed time delay is increased further to values comparable to the cycle period for example, we recover that some long-term modulation is also produced and that a series of bifurcations may be identified. We thus confirm the results of Yoshimura (1978) that significant time delays modify the order of the equations and give solutions which are far from being harmonic in time. However, the purpose of our work was to show that even small delays can also produce similar long-term modulation, provided that those delays vary with the variable (the toroidal magnetic field) itself.

\subsection{The quenching factor}

The analytical study of the reduced model and particularly of the stability of the harmonic solution (described in detail in Jouve, Proctor \& Lesur 2010) shows that the strength of the quenching term also plays a significant role in the model. In particular, the threshold for the destabilisation of the harmonic solution is strongly modified when $B_{0}$ (or equivalently $\lambda$, see Eq. 2.1 and 2.3) is varied. As a result, for the threshold delay at which the harmonic solution with $B_{0}=10^{4}$ loses stability, the solution with $B_{0}=10^{3}$ 

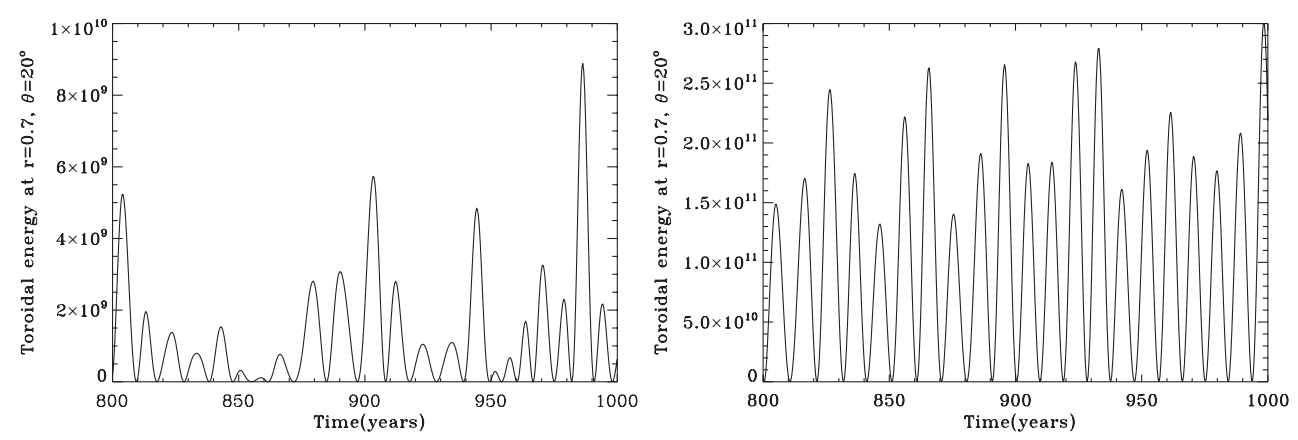

Figure 5. Influence of the quenching strength on the long-term modulation of the cyclic activity. The left panel corresponds to a value of $B_{0}=8 \times 10^{3}$ and the right panel to

$$
B_{0}=5 \times 10^{4} .
$$

will still be stable and the one with $B_{0}=10^{5}$ will already have undergone the period doubling bifurcations leading to chaos.

Similar results are found with the $2 \mathrm{D}$ model when $B_{0}$ is varied, at least qualitatively. Figure 5 shows the time evolution of the toroidal magnetic energy in cases where $B_{0}=$ $8 \times 10^{3}$ (left panel) and $B_{0}=5 \times 10^{4}$ (right panel). Indeed, when the non-linearity is high (corresponding to a value of $B_{0}=8 \times 10^{3}$ ), the modulation of the cycle amplitude is very strong, the energy of the strongest peaks reaching about 45 times the value for the weakest cycles. Another striking point is that not only is the amplitude modulated but the cycle period also seems to vary from one cycle to another. In particular, the very weak cycles between $t=950$ and $t=970$ exhibit periods of only $60 \%$ of the cycle peaking at $t=880$ for instance. On the contrary, when $B_{0}$ is higher, the quenching term is less efficient and the solution seems much less modulated, the ratio between the highest peaks and the weakest in this particular time interval being of order 2 . These results can be explained by the presence of the delays in the non-linear quenching term. Indeed, not only is the poloidal regenerated by the delayed toroidal field, it is also saturated by this same field. This particular feedback is thus probably responsible for the differences in the modulation of the activity even when the delay is kept identical, and thus small compared to the period.

\subsection{Adding a tachocline $\alpha$-effect}

We may now introduce a variation on the model itself by adding an $\alpha$-effect in the tachocline. Indeed, there is no clear reason why a regeneration term for poloidal fields should only be confined at the solar surface, the more "classical" $\alpha$-effect due to helical motions in the convection zone might also be responsible for the stretching of toroidal field and its conversion into a poloidal component. Some studies (Mason, Hughes \& Tobias 2002, Mann \& Proctor 2009) have shown that even a weak $\alpha$-effect at the base of the convection zone might be dominant over the BL source term. For example, Mann \& Proctor (2009) have shown that a tachocline effect of 3 orders of magnitude less than a surface source term still controlled the behaviour of the magnetic field in the case where a non-local BL effect is considered. It is thus natural to think that adding a tachocline $\alpha$-effect, however weak, could modify the effect of the time delays on the long-term modulation. Indeed, the time delays are only present to take into account the spatial segregation between the source terms of both components of the magnetic field, there is thus no reason to introduce a delay for a tachocline $\alpha$-effect, which acts locally on the toroidal field. Moreover, this process being easily dominant on the field evolution, we might see it as a stabilising effect. 
We have thus introduced an additional $\alpha$-effect concentrated in a thin layer between 0.68 and 0.78 in the most perturbed model we had ( 14 days-delay on $5 \times 10^{4} \mathrm{G}$ fields). Our preliminary calculations, with a tachocline $\alpha$-effect about 2 orders of magnitude smaller than the BL source term (and thus supposed to be dominant according to Mann $\&$ Proctor results) do not seem to show any stabilisation of the cycle modulation. The evolution of the toroidal magnetic field still seems to be chaotic, with some bursts of activity coexisting with periods of grand minima. This might be understood by the fact that if the time delays in the BL term have a slight effect on the modulation of the toroidal field amplitude, it may be in fact amplified by the presence of the tachocline $\alpha$-effect which will act on the modulated toroidal field to produce the new poloidal flux. As a consequence, the small time delays and the weak BL source term may still be significant enough to produce some modulation which will in turn be amplified by the additional instantaneous (or undelayed) $\alpha$-effect. Moreover, Mann \& Proctor (2009) showed that adding a fast meridional flow had the tendency to reduce the efficiency of the tachocline $\alpha$-effect, suggesting that we may still be dominated by the BL source term in our particular case. This still needs some more investigation, especially if we want to consider a distributed $\alpha$-effect instead, which may also be subject to delays.

\section{Conclusions}

Delayed differential equations appear to have some interesting properties on the dynamical system point of view. In the particular case of the mean-field dynamo equations, time delays can be related to the advection time by the meridional flow and the rise time of toroidal structures from the base of the convection zone to the solar surface. Both can be responsible for long-term modulation of the cyclic magnetic activity. The effect of time delays due to the buoyant rise of flux tubes is of particular interest since those delays are thought to be small compared to the cycle period (about a few percents typically). However, the fact that those delays are dependent on the magnetic field makes the governing equations belong to the class of state-dependent delayed partial differential equations. Their properties and their differences with fixed delayed equations are still not completely understood and in our particular case, we showed that they indeed produce solutions with various temporal evolutions.

A considerable step forward would obviously be to develop a self-consistent global model with buoyant toroidal structures built up and making their way from the base of the convection zone where they become unstable to the photosphere where they create active regions. Unfortunately, this has not been achieved yet due to numerous physical and numerical difficulties. In the mean time, we have shown here that the combination of 3D MHD models simulating a particular step of the dynamo cycle, 2D mean-field calculations and reduced models help us to progress on the influence of isolated physical processes on the magnetic cycle. In particular, we have shown that they can even help reproducing a feature of the solar cycle (its variability) which was not present in the standard model before. We will continue to develop these ideas in future work.

\section{References}

Bushby, P. J., 2006, Mon. Not. R. Astron. Soc., 371, 772-780

Charbonneau, P. \& Dikpati, M. 2000, Astrophysical Journal, 543, 1027

Charbonneau, P., St-Jean, C. \& Zacharias, P., 2005, Astrophysical Journal, 619, 613

Hoyng, P. 1988, Astrophysical Journal, 332, 857

Jouve, L. \& Brun, A. S. 2009, Astrophysical Journal, 701, 1300 
Jouve, L., Proctor, M. R. E. \& Lesur, G. 2010, Astronomy \& Astrophysics, 519, A68

Mann, P. D. \& Proctor, M. R. E. 2009, Mon. Not. R. Astron. Soc., 399, 99

Mason, J., Hughes, D. \& Tobias, S. 2002, Astrophysical Journal, 580, L89

Moss, D. \& Brooke, J., 2000, Mon. Not. R. Astron. Soc., 315, 521-533

Ossendrijver, A. J. H. \& Hoyng, P. 1996, Astronomy \& Astrophysics, 313, 959

Proctor, M. R. E. 1977, Journal of Fluid Mechanics, 80, 769

Rempel, M., 2006, Astrophysical Journal, 647, 662

Tobias, S. M. 1997, Astronomy \& Astrophysics, 322, 1007

Weiss, N. O. \& Tobias, S. M. 2000, Space Science Reviews, 94, 99

Wilmot-Smith, A. L., Nandy, D., Hornig, G. \& Martens, P. C. H. 2006, Astrophysical Journal, 652,696

Yeates, A. R., Nandy, D. \& Mackay, D. H. 2008, Astrophysical Journal, 673, 544

Yoshimura, H. 1978, Astrophysical Journal, 226, 706

\section{Discussion}

T. Rogers: The time delay implied by the mean field models to get aperiodic modulation was around $10 \%$ of the cycle period, but the rise time implied by the simulations was a few days. This is a big difference, how do you reconcile the two?

L. Jouve: In the 3D model, the field strengths considered are quite high (more than $2 \times 10^{5} \mathrm{G}$ in order to rise radially and not parallel to the rotation axis). We thus get rise times which are very short (about a few days). However, the time delay depends on the inverse of $|B|^{2}$ and thus if a $10^{5} \mathrm{G}$ field is delayed by 5 days, a $10^{4} \mathrm{G}$ field will be delayed by about 16 months, which corresponds to the values chosen for the $2 \mathrm{D}$ model. It is more difficult to relate thoses values to the reduced model which mostly serves illustrative purposes. Nevertheless, a fixed delay of $10 \%$ of the cycle is still inefficient to produce long-term modulation, so can still be considered small.

G. VASIL: Does the nonlinear time delay needs to be respect to the buoyancy terms or could it just as easily be attached to some other process in the system?

L. Jouve: The non-linear term in the time delay (proportional to $1 / B^{2}$ ) was chosen to represent the magnetic buoyancy effects. Other formulations (with various non-linear functions of the magnetic field) were not tested but the intensity of this term is here shown to have a significant effect on the threshold for destabilization of the periodic solution.We could imagine representing the meridional flow by a time-delay and since concentrations of strong fields are known to modify this flow, this time delay could also be non-linearly dependent on $B$. This nonlinear delay would thus be linked to the transport by meridional flows instead of buoyancy. This has not yet been tested but could be interesting to consider.

S. Tobias: Do you see signs of a secondary frequency and if so, how does it depend on the delay?

L. Jouve: The spectra show that it is essentially smaller frequencies (or larger periods) which are excited when the delay is increased. In our most perturbed case, particular periods of about 20 years and 40 years seem to appear, possibly indicating previous period doublings which led to the observed chaotic behaviour. 